Среднеотдаленные результаты чрескожных коронарных вмешательств с имплантацией стандартного металлического стента "Ephesos II" у больных ишемической болезнью сердца

\author{
Т.А. Батыралиев ${ }^{1}$, А.В. Фетцер ${ }^{*}$ А.В. Преображенский ${ }^{2}$, А. Кочак ${ }^{1}$ Ю.Н. Беленков ${ }^{3}$
}

Международная исследовательская группа по клинической и интервенционной кардиологии:

${ }^{1}$ Медицинский центр им. Сани Конукоглы. Газиантеп, Турция; ${ }^{2}$ УМЦ Управления делами Президента РФ. Москва, Россия; ${ }^{3}$ Московский государственный университет имени М.В. Аомоносова

\title{
Middle-term results of percutaneous coronary intervention with standard metallic stent "Ephesos II" implantation in patients with coronary heart disease
}

\author{
T.A. Batyraliev ${ }^{1}$, D.V. Fetzer*, D.V. Preobrazhenskyi , A. Kochak ${ }^{1}$, Yu.N. Belenkov ${ }^{3}$ \\ International Work Force on Clinical and Interventional Cardiology: ${ }^{1}$ Sani Konukoglu Medical Centre. \\ Gaziantep, Turkey; ${ }^{2}$ RF President's Administration Medical Centre. Moscow, Russia; ${ }^{3}$ M.V. Lomonosov \\ Moscow State University
}

Цель. В нерандомизированном исследовании оценить эффект и безопасность выполнения чрескожных коронарных вмешательств (ЧКВ) с имплантацией стента “Еphesos II” в среднем в течение 9 мес.

Материал и методы. В исследование был включен 41 больной. Пациенты проходили интервенционное лечение в Медицинском центре им. Сани Конукоглы, Газиантеп, Турция.

Результаты. Непосредственный ангиографический успех вмешательств был достигнут у 100 \% больных. За 9 мес. наблюдения доля выживших больных без рестеноза и повторной реваскуляризации составила 77,6 \%. Ангиографический контроль через 9 мес. был выполнен у 95,1 \% больных. Индекс потери диаметра сосуда $=0,32 \pm 0,25$. Рестеноз был выявлен у $22,4 \%$ больных. Во всех случаях рестеноза внутри стента были успешно выполнены повторные ЧКВ. К 9 мес. наблюдения доля выживших больных без выраженных сердечных осложнений и событий составила 70,3\%.

Заключение. Таким образом были получены хорошие ближайшие и среднеотдаленные результаты лечения больных с использованием ЧКВ и с имплантацией стандартного металлического стента "Ephesos II".

Ключевые слова: ИБС, чрескожное коронарное вмешательств, тромбоз, осложнения, стандартный металлический стент.

Aim. In a non-randomised study, to assess middle-term (9 months) effectiveness and safety of percutaneous coronary intervention (PCI) with "Ephesos II" stent implantation.

Material and methods. The study included 41 patients, treated at the Sani Konukoglu Medical Centre, Gaziantep, Turkey.

Results. Immediate angiography-confirmed PCI success was achieved in $100 \%$ of the participants. Nine months after the intervention, the percentage of survived patients without restenosis and repeat revascularization was $77,6 \%$. Control angiography at 9 months was performed in $95,1 \%$ of the patients. The mean in-stent late loss was $0,32 \pm 0,25$. Restenosis was observed in $22,4 \%$ of the subjects. In all cases of in-stent restenosis, successful repeat PCI was performed. At 9 months, the proportion of the survived patients without moderate to severe cardiac complications and events reached $70,3 \%$.

Conclusion. This non-randomised study demonstrated good short and middle-term results of PCI with standard metallic stent "Ephesos II" implantation.

ОКоллектив авторов, 2011

e-mail: fettser@gmail.com

['Батыралиев Т.А. - директор департамента кардиологии, Фетцер Д.В. ( ${ }^{*}$ контактное лицо) - сотрудник отделения рентгенохирургических методов диагностики и лечения Областной клинической больницы г Липецка, ${ }^{2}$ Преображенский Д.В. - доцент кафедры кардиологии и общей терапии, 'Кочак А. - сотрудник департамента кардиологии, ${ }^{3}$ Беленков Ю.Н. - проректор]. 
Key words: Coronary heart disease, percutaneous coronary intervention, thrombosis, complications, standard metallic stent.

В настоящее время в интервенционной кардиологии используются стандартные металлические стенты разного дизайна, гибкости, выполненные из различных сплавов. Коронарный стандартный металлический стент "Ephesos II" ("Ephesos II" Coronary Stent Delivery System, Alvimedica Inc., Turkey) имеет европейский сертификат СЕ и успешно используется в ряде стран Европы на протяжении нескольких лет. Этот стент пришел на смену хорошо себя зарекомендовавшему стандартному металлическому стенту Ephesos, результаты имплантации которого были представлены в ранних исследованиях [1-4].

\section{Материалы и методы}

В исследование был включен 41 пациент; лечившийся в 2007г со стабильной стенокардией напряжения (III или IV функциональных классов (ФК) по классификации Канадской ассоциации кардиологов или острым коронарным синдромом (ОКС) без инфаркта миокарда (ИМ) с зубцом Q (Q-ИМ) в Медицинском центре им. Сани Конукоглы, Турция. У этих больных во время коронарной ангиографии (КАГ) был выявлен $\geq 1$ гемодинамически-значимый стеноз ( $>50 \%$ диаметра сосуда) без окклюзии ( $<100 \%)$ в одной или нескольких коронарных артериях (KA). Критериями для исключения больных из исследования были: предшествующее чрескожное коронарное вмешательство (ЧКВ) или коронарное шунтирование (КШ) в анамнезе, первые 7 сут. после острого Q- ИМ. Больные, не переносившие аспирин или клопидогрел, а также с геморрагическим инсультом или желудочно-кишечным кровотечением в течение последних 6 мес. перед ЧКВ также были исключены из наблюдения. Ангиографическими критериями исключения из исследования были стеноз ствола левой KА, трехсосудистое поражение КА, бифуркационное поражение КА, устьевой стеноз передней нисходящей, огибающей или правой КА, должный диаметр сосуда (ДДС) в месте стеноза $<2,5$ мм и ангиографические признаки острого внутрикоронарного тромбоза целевой КА с показаниями для назначения блокаторов гликопротеиновых IIb/IIІа рецепторов тромбоцитов. Все больные давали письменное информированное согласие на участие в исследовании согласно этическому протоколу.

Дизайн стента. “Ephesos II” - это баллон-расширяемый матричный стент с многоячеистым дизайном, созданный из сплава хрома и кобальта по американскому стандарту F562. По сравнению с нержавеющей сталью $316 \mathrm{~L}$, из которой изготавливали стент Ephesos первой генерации, хром-кобальтовый сплав позволил создать более прочный стент с высокой радиальной жесткостью и гибкостью. Номинал диаметров расправленного стента: 2,$5 ; 2,75 ; 3,0 ; 3,5 ; 4,0 ; 4,5$ мм. Длина нерасправленного стента представлена следующими размерами: $8,9,12,15$, $18,20,25,28$ и 32 мм, а профиль колеблется от $2,4 \mathrm{~F}$ на проксимальном конце до $2,7 \mathrm{~F}$ на дистальном. При расправлении стента не происходит его значимого укорочения.
Техника ЧКВ. ЧКВ с имплантацией стента выполнялось, как правило, после предилатации стеноза коронарным баллоном. Раздувание стента проводилось в течение 20-30 сек при номинальном давлении 8 атмосфер. Соотношение диаметра стента "Ephesos II" к должному диаметру сосуда 1:1 достигалось постдилатациями стента под давлением от 10 до 16 атм .

Фармакологическая поддержка при ЧКВ. Всем больным от момента госпитализации в клинику назначали аспирин в дозе 100-325 мг/сут., после выписки всем пациентам был рекомендован его постоянный прием. Клопидогрел назначали за сут до стентирования в дозе 75 мг или давали за 6 ч перед процедурой в дозе 300 мг. После имплантации стента "Ephesos II" больному рекомендовали принимать клопидогрел в течение не $<3$ мес. в дозе 75 мг/сут.

Перед ЧКВ больным вводили нефракционированный гепарин (НФГ) болюсом из расчета 80-100 ЕД/кг массы тела через катетер в аорту. Эффективность гепаринотерапии оценивалась по показателю АЧТВ, которое должно было увеличиться в 2,5 раза от исходного (не $<100$ сек), или по АСТ, которое удлиняли $>300$ сек.

Последующее наблюдение и статистический анализ. Анализ результатов ЧКВ включал 4 конечных точки.

Первая точка исследования - оценивались непосредственные ангиографические результаты ЧКВ (рассчитывалась частота следующих событий: смерть, окклюзия КА с последующим ИМ, кровотечения из артерии-доступа).

Вторая точка - клинические результаты до окончания госпитализации (определялась частота смерти, ИМ, возврата стенокардии и повторной реваскуляризации целевого поражения).

Третьей точкой являлись клинические и ангиографические результаты в сроки до 6 мес. после ЧКВ. Клинические результаты подвергались анализу по наступлению одного из событий или истечению срока наблюдения. Они оценивались по суммарной частоте смерти, последующего ЧКВ или операции КШ, ИМ и возвратной стенокардии, связанной со стентированным сегментом. Ангиографические результаты определялись при контрольной КАГ через 6 мес. или при исследовании в более ранние сроки в связи с рецидивом стенокардии или ИМ. Рассчитывалась частота бинарного рестеноза (повторного сужения стентированной КА на $\geq 50 \%$ по диаметру.

Четвертая точка включала клинические результаты в сроки до 9 мес. после ЧКВ. Они оценивались по суммарной частоте смерти, последующего ЧКВ или операции КШ, ИМ и возвратной стенокардии аналогично наблюдению до 6 мес.

Ангиографический анализ включал в себя определение рентгеноморфологии стеноза КА согласно классификации Американской коллегии кардиологов и Американской ассоциации сердца (АСС/АНА) [5]. Количественный коронарный анализ проводили с помощью системы QuantCor (Siemens, Германия) и включал определение минимального диаметра сосуда (МДС) до стентирования; должного диаметра сосуда (ДДС); \% стеноза (МДС / ДДС) и длины стеноза. Изменения МДС фиксировались сразу после стентирования и через 9 мес. наблюдения. Определялись прирост диаметра: МДС после 
Таблица 1

Исходные клинические характеристики больных

\begin{tabular}{ll}
\hline Клиническая характеристика & ЧКВ \\
$\mathrm{n}=41$ \\
\hline Возраст, лет & $59,3 \pm 11,2$ \\
Мужчины, \% & 75,6 \\
АГ, \% & 63,4 \\
СД, \% & 24,4 \\
Курильщики, \% & 39,0 \\
Гиперхолестеринемия, \% & 29,3 \\
МИ в анамнезе, \% & 4,9 \\
ИМ в анамнезе, \% & 41,5 \\
ОКС, \% & 43,9 \\
\hline
\end{tabular}

Исходные ангиографические характеристики больных

Таблица 2

\begin{tabular}{ll}
\hline Ангиографическая характеристика & ЧКВ на 52 КА сегментах \\
\hline Многососудистые поражения КА, \% & 36,6 \\
Передняя нисходящая КА, \% & 55,8 \\
Огибающая КА, \% & 25,0 \\
Правая КА, \% & 19,2 \\
Стеноз типа А-В1, \% & 63,5 \\
Стеноз типа В2-С, \% & 36,5 \\
Минимальный диаметр сосуда, мм & $0,74 \pm 0,41$ \\
Должный диаметр сосуда, мм & $3,31 \pm 0,43$ \\
Должный диаметр сосуда < 3 мм, \% & $24,4 \%$ \\
Стеноз по диаметру, \% & $77 \pm 11$ \\
Длина стеноза, мм & $14,9 \pm 6,7$ \\
\hline
\end{tabular}

Основные характеристики ЧКВ

Таблица 3

\begin{tabular}{lc}
\hline Характеристика & Показатель \\
\hline Непосредственный успех ЧКВ, \% & 100 \\
Стентирование одной КА, \% & 73,2 \\
Одномоментное стентирование двух КА, \% & 26,8 \\
Средняя длина имплантированного стента, мм & $16 \pm 8$ \\
Должный диаметр сосуда после стентирования, мм & $3,49 \pm 0,53$ \\
Минимальный диаметр сосуда после стентирования, мм & $3,27 \pm 0,40$ \\
Прирост диаметра сосуда, мм & $2,53 \pm 0,49$ \\
\hline
\end{tabular}

стентирования -МДС до стентирования; потеря диаметра: МДС после стентирования -МДС через 9 мес.; итог диаметра: МДС через 9 мес. -МДС до стентирования; индекс потери диаметра: потеря диаметра / прирост диаметра.

Для статистической обработки использовался пакет "Statistica Base for Windows v. 6" (StatSoft Inc, США). Значения представлены как среднее \pm стандартное отклонение. Достоверным считали уровень $\mathrm{p}<0,05$.

\section{Результаты}

Исходные характеристики больных представлены в таблице 1. В настоящем исследовании большинство больных были мужчины (75,6 \%). Артериальная гипертензия (АГ) была зарегистрирована у 63,4 \% пациентов. Каждый четвертый больной $(24,4 \%)$ страдал сахарным диабетом (СД) и получал специфическую терапию per os или инсулин подкожно (п/к). Курильщики соста- вили 39,0 \% от общего количества больных. Каждый третий больной $(29,3 \%)$ имел повышенный уровень холестерина (ХС) в крови (> 5,2 ммоль/л). Инсульт (МИ) в анамнезе был у $2(4,9 \%)$ больных. Менее половины пациентов $(41,5 \%)$ ранее перенесли ИМ, а 43,9 \% больных на момент текущей госпитализации имели клинику ОКС.

Исходные ангиографические характеристики больных отражены в таблице 2. Во время 41 ЧКВ было имплантировано 52 стента "Ephesos II". Гемодинамически-значимые стенозы в двух КА имелись у 36,6 \% больных, у 26,8 \% пациентов эти стенозы подверглись стентированию во время одного ЧКВ. Наиболее часто поражения локализовались в передней межжелудочковой КА (55,8 \%), реже - в огибающей КА $(25,0 \%)$ и правой 
Таблица 4

Клиническое наблюдение в течение 6 мес. после ЧКВ

\begin{tabular}{ll}
\hline Характеристика & Показатель \\
\hline Смерть, \% & 2,4 \\
Острый/подострый тромбоз КА, \% & 0 \\
ИМ, \% & 2,4 \\
МИ, \% & 0 \\
Возвратная стенокардия, \% & 12,5 \\
Рестеноз в сегменте, \% & 15,6 \\
Повторное ЧКВ, \% & 15,6 \\
КШ, \% & 0 \\
\hline
\end{tabular}

Клиническое наблюдение в течение 9 мес после ЧКВ

Таблица 5

\begin{tabular}{ll}
\hline Характеристика & Показатель \\
\hline Смерть от всех причин, \% & 4,9 \\
ИМ, \% & 7,3 \\
МИ, \% & 2,4 \\
Возвратная стенокардия, \% & 17,9 \\
Рестеноз в сегменте, \% & 22,4 \\
Повторное ЧКВ, \% & 22,4 \\
КШ, \% & 0 \\
Частота выраженных сердечных осложнений и событий, \% & 29,7 \\
\hline
\end{tabular}

Таблица 6

Данные ангиографического контроля через 9 мес.

\begin{tabular}{lc}
\hline & 39 больных, 49 сегментов \\
\hline Окклюзия сосуда, $\%$ & 0 \\
Рестеноз в сегменте: & 11,5 \\
локальный (<10 мм), $\%$ & 9,6 \\
диффузный ( $\geq 10$ мм), $\%$ & $3,22 \pm 0,42$ \\
дДС, мм & Количественный коронарный анализ \\
МДС, мм & $2,45 \pm 0,77$ \\
Стеноз по диаметру, $\%$ & $24 \pm 19$ \\
при рестенозе & $78 \pm 13$ \\
без рестеноза & $16 \pm 14$ \\
Потеря диаметра сосуда, мм & $0,82 \pm 0,58$ \\
Итог диаметра сосуда, мм & $1,71 \pm 0,80$ \\
Индекс потери диаметра сосуда, мм & $0,32 \pm 0,25$ \\
при рестенозе, мм & $0,93 \pm 0,24$ \\
без рестеноза, мм & $0,23 \pm 0,12$ \\
\hline
\end{tabular}

КА (19,2 \%). Стенозирование КА по диаметру в среднем составило $77 \pm 11 \%$ при средней длине стеноза 14,9 $\pm 6,7$ мм.

Основные характеристики ЧКВ представлены в таблице 3. В исследовании все 52 стента "Ephesos II” были успешно имплантированы больным без развития диссекций типа D-F, острого закрытия сосуда или феномена "no reflow". Таким образом, непосредственный ангиографический успех имплантации стентов составил $100 \%$.

В первые сут после ЧКВ у 1 (2,4 \%) больного развилось кровотечение, которое было разрешено консервативно наложением тугой давящей повязки. Другие периферические или выраженные сердечные и цереброваскулярные осложнения и события на госпитальном этапе отсутствовали. За время последующего наблюдения в сроки до 6 мес. умер 1 больной в результате ОИМ (тромбоз ствола левой КА) (таблица 4). При повторной КАГ до 6 мес. рестеноз был выявлен в 15,6 \% стентированных сегментах. В 2 случаях рестеноз не сопровождался возвратной стенокардией. Согласно рекомендациям [5-7] во всех случаях рестеноза были успешно выполнены повторные реваскуляризации с помощью ЧКВ. Повторные вмешательства закончились успешно у всех больных.

Таким образом, в настоящем исследовании суммарная частота рестеноза, возвратной стенокардии, летальных осложнений и последующих ревас- 
куляризаций за 6 мес. наблюдения после ЧКВ с имплантацией стента "Ephesos II" была 18,0\%, а доля больных без выраженных сердечных осложнений и событий, соответственно, составила $82,0 \%$. Проведенный количественный коронарный анализ через 6 мес. после ЧКВ не выявил ни одной тотальной окклюзии.

За время последующего клинического наблю-

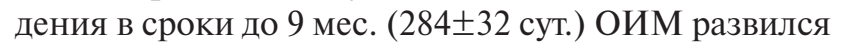
у 3 больных, 1 из которых умер. Еще 1 больной умер в результате повторного МИ через 7 мес. после ЧКВ. Частота рестеноза за 9 мес. в исследовании составила $22,4 \%$, и во всех случаях рестеноза было повторно выполнено ЧКВ. Выраженные сердечные осложнения и события за 9 мес. были зарегистрированы у 29,7 \% больных (таблица 5). Результаты ангиографического контроля представлены в таблице 6.

\section{Обсуждение}

За несколько десятилетий клинического применения сменилось несколько поколений коронарных стентов [8, 9]. В конце 80-х - начале 90-х годов исследователи доказали, что использование стентов не приводит к росту острых и подострых осложнений в сравнении с баллонной ангиопластикой (БАП) [10-12]. Также установлено, что при стентировании значительно снижается частота рестеноза в течение 6 мес. после ЧКВ [13]. Исследователи выяснили, что в реальной практике проволочные стенты не обладают какими-либо преимуществами перед другими чрескожными методами в отношении отдаленных выраженных сердечных осложнений и событий [9]. Основными устройствами с доказанными преимуществами оказались матричные/тубулярные стенты [13-16]. Однако при использовании стентов удалось лишь снизить частоту рестеноза, но ни одна из конструкций не могла устранить его совсем. Новой вехой в интервенционной кардиологии стали стенты, выделяющие лекарства, представленные в 2001г $[17,18]$. Их использование резко снизило частоту рестеноза, что позволило им завоевать популярность и несколько потеснить стандартные металлические стенты [19,20]. Однако конструкция стента по-прежнему играет важное значение. Несмотря на ряд успешно проведенных ранее исследований в 2004г компания "Boston Scientific" была вынуждена в срочном порядке отозвать 40 тыс. стентов, выделяющих лекарства, “TAXUS" и 60 тыс. стандартных металлических стентов "EXPRESS" в связи с обнаруженными дефектами в системе стента. Дефект не позволял сдуть коронарный баллон и удалить его после имплантации стента, что привело к ряду осложнений, включая летальные исходы. Таким образом, особенности дизайна и конструкции стента остаются актуальными и в эру стентов, выделяющих лекарства. Применение стента, обеспечивающего стабильные результаты, дает интервенционному кардиологу возможность прогнозировать отдаленные исходы. Знать реальные возможности стента крайне важно, чтобы оценивать влияние его дизайна на тромбоз и гиперплазию неоинтимы в месте имплантации [15,221-25].

В настоящем исследовании стандартный металлический стент “Ephesos II" был имплантирован в стенозы различной сложности без развития острого или подострого тромбоза в стенте. При этом частота бинарного рестеноза в сегменте за 9 мес наблюдения составила 22,4 \%. Такая частота рестеноза позволяет отнести стент "Ephesos II” к лучшим стандартным металлическим стентам, используемым в настоящее время [15,22, 24,25]. Одной из причин низкой частоты рестеноза может являться большой средний должный диаметр сосуда $(3,31 \pm 0,43$ мм). Однако не стоит забывать, что большая доля поражений имела высокий риск рестеноза: 36,5 \% стенозов были типа В2 или С, a у 24,4 \% больных должный диаметр КА был $<3,0 \mathrm{MM}$.

Наблюдение в сроки до 9 мес. показало, что после ЧКВ с имплантацией стента "Ephesos II" отмечается приемлемая частота выраженных сердечных осложнений и событий. Невысокая смертность по сердечным причинам $(2,4 \%)$ связана со своевременным лечением ишемической болезни сердца. > 22 \% больных за время наблюдения перенесли повторную реваскуляризации миокарда, но во всех случаях удалось избежать КШ - всем больным было выполнено повторное ЧКВ.

Ограничения исследования. В настоящем исследовании не предполагалось прямого сравнения стента "Ephesos II" с другими стандартными металлическими стентами или стентами, выделяющими лекарства. В исследование было включено относительно небольшое количество пациентов.

\section{Выводы}

Таким образом, ЧКВ с имплантацией стандартного металлического стента “Ephesos II" позволяет получить хорошие ближайшие и среднеотдаленные результаты лечения при нативных поражениях в КА. Исход имплантации стента "Ephesos II" за 9 мес. наблюдения оказался благоприятным без выраженных сердечных осложнений и событий у 70,3 \% больных. 


\section{Литература}

1. Uyan C, Akdemir R, Gunduz H, et al. Immediate and six months clinical and angiographic results of intracoronary Ephesos stent implantation: the "Ephesos-1" study. Acta Cordial 2005; 60(1): 185-90.

2. Беленков Ю.Н., Самко А.Н., Батыралиев Т.А. и др Двухлетние результаты после имплантации металлического стента "Ephesos" у пациентов с коронарной болезнью. Тер архив 2005; 9: 49-52.

3. Pershukov I, Batyraliev T, Samko A, et al. Results of "Ephesos" coronary stent implantation in patients with unstable or stable angina. Eur J Gen Med 2004; 1: 19-25.

4. Батыралиев Т.А., Самко А.Н., Першуков И.В. и др. Клинические и ангиографические результаты применения стента Ephesos у больных атеросклерозом коронарных артерий. Кардиология 2002; 2: 4-7.

5. King SB, Aversano T, Ballard WL, et al. ACCF/AHA/SCAI 2007 Update of the Clinical Competence Statement on Cardiac Interventional Procedures. Circulation 2007; 116: 98-124.

6. Balcon R, Beyar R, Chierchia S, et al. for the Study Group of the Working Group on Coronary Circulation. Recommendations on stent manufacture, implantation and utilization. Eur Heart J 1997; 18: 1536-47.

7. Eeckhout E, Wijns W, Meier B, Goy JJ on behalf of the members of the Working Group on Coronary Circulation of the European Society of Cardiology. Indications for coronary stent placement: the European view. Eur Heart J 1999; 20: 1014-9.

8. Serruys PW, Kutryk MJB. Handbook of coronary stents. Third edition. Martin Dunitz 2000; p.424.

9. Бабунашвили А.М., Иванов В.А., Бирюков С.А. Эндопротезирование (стентирование) венечных артерий сердца. Издательство АСВ, Москва 2000; 704 с.

10. Sigwart U, Puel J, Mirkovitch V, et al. Intravascular stents to prevent occlusion and restenosis after transluminal angioplasty. N Engl J Med 1987; 316: 701-6.

11. Батыралиев Т.А., Фетцер Д.В., Преображенский Д.В., Сидоренко Б.А. Проблема осложнений при чрескожных коронарных вмешательствах. Часть I. Характер и частота осложнений. Кардиология 2008; 48(1): 81-7.

12. Sigwart U, Urban P, Golf S, et al. Emergency stenting for acute occlusion after coronary balloon angioplasty. Circulation 1988; 78: 1121-7.

13. Serruys PW, de Jaegere P, Kiemeneij F, et al. A comparison of balloon-expandable-stent implantation with balloon angioplasty in patients with coronary artery disease: Benestent Study Group. N Engl J Med 1994; 331: 489-95.

14. Baim DS, Levine MJ, Leon MB, et al. Management of restenosis within the Palmaz-Schatz coronary stent: the U.S. multicenter experience - the U.S. Palmaz-Schatz Stent Investigators. Am J Cardiol 1993; 71: 364-6.

15. Antoniucci D, Bartorelli A, Valenti R, et al. Clinical and Angiographic Outcome After Coronary Arterial Stenting With the Carbostent. Am J Cardiol 2000; 85: 821-5.

16. Boland JL, Corbeij HAM, Van Der Giessen W, et al. Multicenter evaluation of the phosphorylcholinecoated biodivYsio stent in short de novo coronary lesions: The SOPHOS study. Int J Cardiovasc Intervent 2000; 3: 215-25.

17. Батыралиев Т.А., Першуков И.В. Обзор клинических исследований по ишемической болезни сердца и ее инвазивному лечению. Кардиология 2002; 5: 68-70.

18. Rensing BJ, Vos J, Smits PC, et al. Coronary restenosis elimination with a sirolimus eluting stent. First European human experience with 6-month angiographic and intravascular ultrasonic follow-up. Eur Heart J 2001; 22: 2125-30.

19. Беленков Ю.Н., Батыралиев Т.А., Першуков И.В., Самко А.Н. Инвазивная кардиология - фокус на рестеноз. Часть II. Кардиология 2002; 11: 68-72.

20. Жамгыргиев Ш.Т., Фетцер Д.В., Першуков И.В. и др. Клиническое значение стентов с лекарственным покрытием. Тер архив 2007; 9: 79-84.

21. Zidar JP, Fry E, Lambert C, et al. The VENUS trial: A multicenter registry of the Cordis BX Velocity stent. Am J Cardiol 2000; 86: suppl.8A: 17i.

22. Boland JL, Corbeij HAM, Van Der Giessen W, et al. Multicenter evaluation of the phosphorylcholinecoated Biodivysio stent in short de novo coronary lesions: The SOPHOS study. Int $\mathbf{J}$ Cardiovasc Intervent 2000; 3: 215-25.

23. Kornowski R, Hong MK, Tio FO, et al. In-stent restenosis: contributions of inflammatory response and arterial injury to neointimal hyperplasia. JACC 1998; 31: 224-30.

24. Rogers C, Edelman ER. Endovascular stent design dictates experimental restenosis and thrombosis. Circulation 1995; 91 : 2995-3001.

25. Serruys PW, van Hout B, Bonnier H, et al. Randomized comparison of implantation of heparin-coated stents with balloon angioplasty in selected patients with coronary artery disease (Benestent II). Lancet 1998; 352: 673-81.

Поступила 14/05-2009 